\title{
Elaboración del sistema de gestión ambiental basado en buenas prácticas de manufactura en una empresa de jugos y licores
}

\section{Elaboration of the environmental management system based on good manufacturing practices in a juice and liquor company}

Tamayo Freddy

Coral Katty

Universidad Internacional SEK Ecuador, Ecuador

Rodríguez Ana

Oviedo Jorge E

Universidad de Cantabria, España

Autor de correspondencia: katty.coral@uisek.edu.ec, katty_coral@ hotmail.com

Fecha de recepción: 10 de Agosto 2017 - Fecha de aceptación: 16 de Marzo de 2018

Resumen: La implementación de Buenas Prácticas de Manufactura dentro de un Sistema de Gestión Ambiental innova la Planificación Ambiental, englobando parámetros que buscan el correcto uso de recursos y materias primas con la finalidad de que estos sean optimizados dentro de los procesos productivos a nivel industrial. La empresa, tomada como modelo, se dedica al envasado de jugos y licores; ha sido elegida debido a su necesidad de optimizar procesos, mejorar rentabilidad y velar por el bienestar de sus empleados. El alcance del proyecto está determinado por el número de empleados y los procedimientos productivos con los que cuenta la empresa tanto para el envasado de jugo como de licor, así como por los desechos generados tanto sólidos como efluentes líquidos y gaseosos de los diferentes procesos operativos de la empresa, esto conlleva a un manejo adecuado e integrado que tiene como finalidad establecer procedimientos con criterios de mejora continua; adicionalmente propone el buen manejo de recursos y materia prima con fines de sostenibilidad. Los objetivos que se plantean en el proyecto son establecer un diagnóstico del sistema productivo de la empresa basado en la normativa vigente de buenas prácticas de manufactura del Ministerio de Industrias de Ecuador, este diagnóstico permitirá concatenar a estos requerimientos con el manejo ambiental de la empresa. El sistema de gestión ambiental basado en buenas prácticas de manufactura contempla una estructura conformada por planes, programas, subprogramas, manuales y procedimientos, lo que lo hace comprensible para cualquier persona que desee ponerlo en práctica, de tal forma que con una mínima capacitación por parte de la empresa, se garantice la continuidad y optimización del sistema implantado. La implementación de sistemas de gestión ambiental basados en buenas prácticas de manufactura no solamente minimiza los impactos ambientales generados por las actividades productivas, si no que se controla la generación de los efectos ambientales en el origen, facilitando el tratamiento y la disposición final de los efluentes, residuos generados en el proceso y optimización de recursos.

Palabras Claves: industria; contaminación; política ambiental; economía; alimento; riesgo

Abstract: The implementation of Good Manufacturing Practices within an innovative Environmental Management System Environmental Planning, include parameters seeking the proper use of resources and raw materials in order that these are optimized in production processes. The company was chosen as a model, -dedicated to the packaging of juices and liquors have been selected because of their need 
to optimize processes, improve profitability and ensure the welfare of its employees. The project scope is determined by the number of employees and production with which the company has procedures as well as solid wastes, liquid effluents and gas generated from the various operating processes of the company, this requires proper handling and integrated procedures intended to establish criteria for continuous improvement and good management of resources and raw materials for sustainability. The objectives of the project are to establish a diagnosis of productive enterprise system based on the existing rules of good manufacturing practices of the Ministry of Industry of Ecuador. This diagnosis will allow concatenating these requirements with the environmental management of the company. The environmental management system based on good manufacturing practices consists of plans, programs, subprograms, manuals, and procedures, should be understandable to anyone who wishes to implement structure, and with minimal training by the company, the continuity of the proposed system is guaranteed. The implementation of environmental management systems based on good manufacturing practices minimizes environmental impacts generated by productive activities, and also controls the generation of environmental effects at the source-origin, facilitating the treatment and disposal of effluents and waste generated in the process.

Key words: industry; pollution; environmental politics; economy; food; risk

\section{Introducción}

La implementación de Buenas Prácticas de Manufactura BPM dentro de un Sistema de Gestión Ambiental en la industria alimenticia, basa su importancia en el hecho que abarca parámetros que buscan el correcto uso de recursos y materias primas con la finalidad de que estos sean optimizados dentro de los procesos productivos. (Torres, 2012). La parte ambiental es un aspecto fundamental dentro del sistema de gestión y de buenas prácticas de manufactura, ya que un control y mejoramiento de procesos en cuanto al producto final, generará como resultado desechos y efluentes menos complicados en su tratamiento, pero que igualmente deben ser gestionados adecuadamente (Ministerio de Fomento, España, s.f.).

Según Díaz \& Uría (2009) los antecedentes se remontan a 1906, en Estados Unidos, cuando se creó el Federal Food \& Drugs Act (FDA), esta federación fue creada principalmente para el control de los productos perecibles, ya que los problemas de salud estaban ligados directamente con la forma de alimentación de la población de ese país, creando así políticas públicas que conllevaron a la creación de entidades de control sobre los alimentos producidos dentro de los estados americanos.

En el Ecuador la implantación de BPM, fue propuesta como: "Reglamento de Buenas Prácticas para alimentos procesados. Resolución ARCSA-DE-067-2015-GGG“, por la Dirección Ejecutiva de la Agencia Nacional de Regulación, Control y Vigilancia Sanitaria. Se expidió en Quito D.M., a los 21 días de diciembre de 2015 (Ministerio de Industrias y Productividad, 2016). Existe una normativa vigente en relación a Buenas Prácticas de Manufactura, el Ministerio de Industrias indica que en la actualidad en el Ecuador estas medidas son obligatorias en la industria alimenticia, donde existen discrepancias con respecto a que la elaboración de licor forme parte de esta industria, al igual que el embotellado de estos productos, debido a que las embotelladores no producen el producto, es decir no hay procedimiento productivo justificado, por lo que la materia prima en sí es el producto, sin embargo, en vista de que la empresa objeto de estudio produce adicionalmente jugos, se la considera como industria alimenticia. 
Al igual que las BPM, los sistemas de gestión ambiental no son requerimientos obligatorios, sin embargo, las entidades de control han propuesto varias alternativas con respecto a la implementación de este tipo de medidas a nivel industrial, de manera que las empresas puedan ser reconocidas en sus productos por la implementación de estas medidas en sus procesos, un ejemplo es el caso del sello verde, el cual crea un prestigio adicional a la marca del producto y por ende al fabricante de dicho producto (Ministerio de Industrias y Productividad, 2016).

La asepsia en la elaboración de productos alimenticios, es de suma importancia en todos los procesos productivos. Las buenas prácticas de manufactura buscan el mejoramiento continuo, no solamente de los procedimientos productivos de la empresa, si no que influyen directamente en el personal para que el sistema operacional funcione, dando como resultado el uso correcto de los recursos disponibles, el mejoramiento de calidad del producto final y un manejo sustentable tanto de materias primas como de desechos sólidos y efluentes, resultado de los procesos productivos (Torres, 2012). El envasado de licores es un sector que se encuentra categorizado dentro de la industria alimenticia, por lo cual la regulación, en cuanto a calidad se refiere, es sumamente estricta, ya que si bien el licor no es considerado como un producto de primera necesidad, si es de los que poseen altos porcentajes de demanda a nivel mundial. Por esta razón se debe tener un control de los procesos de envasado, almacenamiento y distribución de los productos mediante la implementación de medidas operativas, a través de la creación de procedimientos y programas vinculados a la mejora continua y funcionamiento óptimo de todo el proceso productivo.

Los procedimientos cumplen el Ciclo de Mejora Continua de Deming, desde su arribo como materia prima, pasando por los procedimientos de elaboración, hasta su deposición final como desecho. Esta gestión se llevará acabo tanto técnica como administrativamente, desde las materias primas, hasta la distribución del producto, con la finalidad de que este mantenga sus propiedades en buen estado y no se genere una perdida para la empresa. La mejora continua se aplicará adicionalmente a los procesos productivos, aumentando su rentabilidad y minimizando su impacto al ambiente. Este ciclo permite a la empresa reducir costos de producción, ya que al crear procedimientos que ayuden a controlar el sistema productivo se reducen pérdidas de materiales y productos. Adicionalmente, es importante hablar de tiempos de producción ya que al crear un sistema optimizado y eficiente, el tiempo de producción decrece, de tal manera que se pueda generar un alcance productivo mucho más amplio del que se tenía implantado previamente dentro de la empresa (Ministerio de Fomento, España, s.f.).

La Empresa en estudio, se encuentra en funcionamiento desde hace 31 años en el sector de La Armenia aproximadamente a 500 metros del Parque Protegido de La Armenia. Tiene linderos con un parque recreativo, una casa y una fábrica. Se dedica al envasado de licor y de jugo de pulpa, siendo los productos de la casa los licores: Ron, Tequila, Vodka, Whisky. Mientras que el envasado de jugos es realizado para empresas que deseen las prestaciones de este tipo de servicios, esto es importante ya que indica que la empresa problema, está en capacidad de regirse a parámetros de manufactura impuestos por sus clientes, lo que garantiza un mayor compromiso con respecto a estándares de calidad.

La elaboración de los diferentes productos de la empresa se realiza por lotes (tipo Batch), es decir se efectúa la producción en base a los pedidos de los clientes, lo cual determina una producción intermitente, ya que lo picos más altos de producción se encuentran en los meses de 
octubre, noviembre y diciembre, por la mayor demanda debido a las festividades que se presentan en estas fechas.

La empresa cuenta con 25 empleados que cumplen funciones en el área operativa de los sistemas de producción de la fábrica embotelladora, cuatro empleados que se encuentran en el área administrativa que consta de la administración general, marketing y ventas de los productos generados por la empresa. Se trabaja en un horario de ocho horas, que laboran de ocho de la mañana a cinco de la tarde, con una hora de almuerzo. La producción, en ciertos casos, requiere que los empleados, especialmente del área de producción, tengan una jornada laboral en el fin de semana.

Dentro de la empresa, la implementación de un sistema de gestión ambiental y buenas prácticas de manufactura, va a generar un valor agregado al producto final, en el cual no se toma en cuenta, al momento de negociar la base del precio de venta, el producto en sí, si no que se mejora el precio de venta en función de un concepto integrado de calidad, de esta manera se reduce el costo total de producción, adquiriendo una amplia apertura en el mercado, ya que el marketing del producto no solamente se basará en la calidad del mismo, si no en el cuidado ambiental, el control de los procesos de producción, y sobre todo la optimización de recursos, lo que redundará en el mejoramiento continuo y la sostenibilidad de la empresa, garantizando la fuente de ingresos de los empleados y empleadores, así como su calidad de vida. (Ministerio de Fomento, España, s.f.). Dentro de la empresa modelo, no se ha implementado un sistema de gestión ambiental en el tiempo de vida de la empresa, así como tampoco se han establecido buenas prácticas de manufactura en los procesos.

El presente trabajo pretende innovar el enfoque tradicional de los sistemas de gestión ambiental, mediante la utilización de buenas prácticas de manufactura como herramienta de producción más limpia, estas son técnicas que permiten definir el estado ambiental tanto de un proceso como de un producto, además de apoyar estrategias y sistemas de tipo ambiental, que tienen como objetivos el diseño, verificación e implantación de un sistema ambiental, además de facilitar la toma de decisiones tanto de tipo administrativo como productivo.

Las buenas prácticas de manufactura, tradicionalmente no han sido contempladas en la implementación de sistemas de gestión ambiental, sin embargo para la industria alimenticia y ante la obligatoriedad de cumplimiento del Reglamento del ARCSA 067 - 2015, esta se constituye en una unidad de análisis de bajo costo, control de contaminantes en el origen y mejoramiento de la cadena de producción, conllevando al mejoramiento de los parámetros ambientales, seguridad y salud ocupacional y de calidad en los productos fabricados por la empresa.

El objetivo de la investigación consistió en elaborar un Sistema de Gestión Ambiental basado en Buenas Prácticas de Manufactura en la empresa, a través de un diagnóstico de las fortalezas y debilidades de su sistema productivo con fines de optimización de recursos y sostenibilidad, para ello fue necesario establecer un diagnóstico del sistema productivo de la empresa basado en la normativa vigente de buenas prácticas de manufactura del Ministerio de Industrias de Ecuador, paralelamente se efectúo el diagnóstico ambiental de la empresa en función de los residuos sólidos, líquidos y gaseosos generados en el proceso productivo para establecer Planes y Programas de Manejo Ambiental, complementariamente se diseñaron procedimientos de 
gestión ambiental basados en buenas prácticas de manufactura para la empresa, sustentados en los diagnósticos realizados para conformar el Sistema de Gestión Ambiental de la empresa. El sistema productivo de la empresa se resume en los diagramas de proceso presentados en los gráficos $1 \mathrm{y}$ 2.

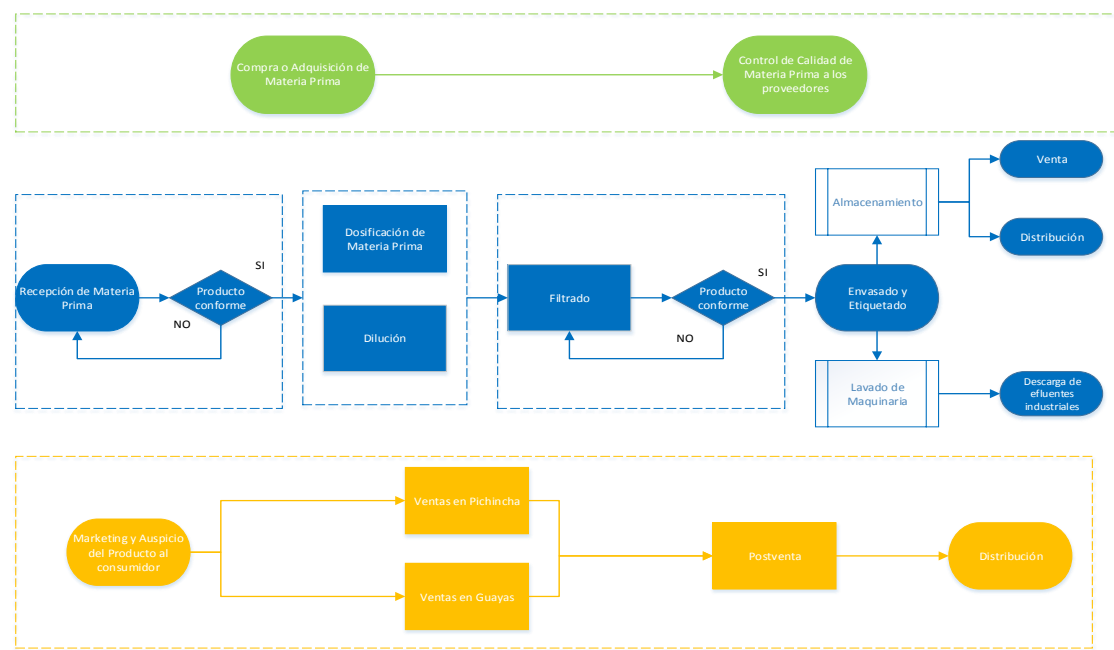

Gráfico 1. Diagrama de proceso de la producción de Licores

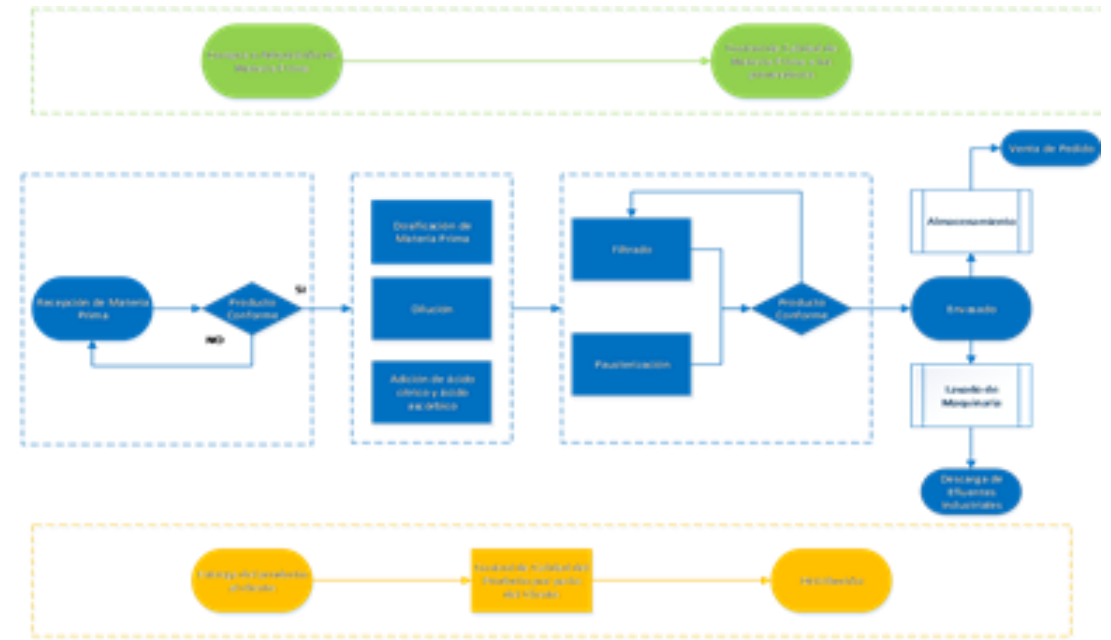

Gráfico 2. Diagrama de proceso de la producción de Jugos

\section{Materiales y métodos}

\section{Investigación Primaria}

Se realizó investigación primaria, mediante páginas de internet, artículos científicos y recopilación de información en las páginas de las instituciones públicas referentes a la implementación del proyecto propuesto, su aplicabilidad, requerimientos y base legal vigente.

Los procesos productivos se levantaron a través de visitas de campo realizadas de manera periódica, esto permitió visualizar, diagnosticar, verificar y descartar información. Adicionalmente se concretaron entrevistas con el encargado del área de producción, parte administrativa y Gerencia General. De esta manera se pudo recopilar información que no era 
factible obtenerla por visualización directa en las visitas de campo. Las entrevistas tuvieron la finalidad de conocer al detalle los diferentes procesos productivos que se emplean para la fabricación de los productos de la empresa, de esta manera se pudo direccionar el diagnóstico para tomar medidas correctivas.

\section{Diagnóstico de Buenas Prácticas de manufactura}

Para realizar el diagnóstico se elaboraron listas de chequeo en función de la Resolución ARCSA-DE-067-2015-GGG, de la Dirección Ejecutiva de la Agencia Nacional de Regulación, Control y Vigilancia Sanitaria, que establece requisitos de buenas prácticas de manufactura. De todos los requisitos establecidos en esta resolución (275), se tomaron en cuenta aquellos vinculados directamente con los procesos productivos, la calidad ambiental y la salud y seguridad ocupacional de la empresa. Utilizando la lista de chequeo generada, se elaboraron matrices de cumplimiento, en las cuales se verificó los hallazgos obtenidos tanto en las visitas de campo como en las entrevistas realizadas para proceder con la evaluación ambiental.

Para la evaluación se tomaron cuatro criterios:

- Conformidad cuando la empresa cumplía con el item entre un 95 a un 100\%

- Conformidad con oportunidad de mejora cuando el cumplimiento de la empresa se encontraba entre un 70 y un $94 \%$.

- No conformidad parcial cuando el incumplimiento se encontraba entre un 50 y un 69\%.

- No conformidad cuando el cumplimiento era inferior al 50\%.

\section{Evaluación Ambiental}

Se realizó la evaluación ambiental para efluentes industriales, emisiones a la atmósfera, ruido ambiental, desechos urbanos e industriales y manejo de combustibles, para lo cual se establecieron todas las leyes vigentes en el país desde el Texto Unificado de Legislación Ambiental, pasando por todos los acuerdos ministeriales de ambiente y seguridad, hasta llegar a las normas INEN.

\section{Evaluación de Seguridad y Salud Ocupacional}

Para la evaluación de seguridad y salud ocupacional se realizaron visitas de campo e inspecciones in situ, donde se pudo verificar de manera visual y fotográfica el cumplimiento de los requerimientos legales establecidos para la empresa, se aplicaron los mismos criterios de cumplimiento que en el acápite anterior.

\section{Resultados y discusión}

\section{Diagnóstico de Buenas Prácticas de manufactura}

De la aplicación de la Resolución ARCSA-DE-067-2015-GGG de la Dirección Ejecutiva de la Agencia Nacional de Regulación, Control y Vigilancia Sanitaria, se generó una lista de chequeo compuesta de 95 items distribuidos como lo indica la tabla 1. 
Tabla 1. Lista de Verificación de Buenas Prácticas de Manufactura de los procesos productivos de la empresa

Lista de verificación de buenas prácticas de manufactura de los procesos productivos de la empresa

Servicios y Facilidades

Item

Disposición de Desechos Líquidos

(1)

Disposición de Desechos Sólidos

Materia Prima

Condiciones de Recepción y Almacenamiento

Operaciones de producción

Condiciones de Producción

Verificación de condiciones de Producción

Métodos de identificación de los Productos

Control de procesos

Envasado, Etiquetado y Empaquetado

Almacenamiento y Distribución de Producto Terminado

Inflamabilidad

Señalética

Personal

Pisos, Paredes, Techos y Drenajes

Ventanas, Puertas y Otras Aberturas

Escaleras, Elevadores y Estructuras Complementarias (rampas, plataformas)

Instalaciones Eléctricas y Redes de Agua

Iluminación

Calidad del Aire y Ventilación

Control de Temperatura y Humedad Ambiental

Instalaciones Sanitarias

$\begin{array}{cc}\text { Controles } & \text { Porcentaje } \\ 7 & 7,4 \\ 2 & 2,1 \\ 7 & 7,4 \\ 4 & 4,2 \\ 4 & 4,2 \\ 4 & 4,2 \\ 4 & 4,2 \\ 1 & 1,1 \\ 1 & 1,1 \\ 3 & 3,2 \\ 9 & 9,5 \\ 8 & 8,4 \\ 1 & 1,1 \\ 2 & 2,1 \\ 3 & 3,2 \\ 5 & 5,3 \\ 2 & 2,1 \\ 1 & 1,1 \\ 2 & 2,1 \\ 2 & 2,1 \\ 3 & 3,2 \\ 1 & 1,1 \\ 5 & 5,3 \\ 5 & 5,3 \\ 6 & 6,3 \\ 2 & 2,1 \\ 1 & 1,1 \\ 95 & 100,0 \\ & \\ & \end{array}$

Equipos y utensilios

Higiene y medidas de protección

Comportamiento del personal

Prohibición de acceso a determinadas áreas

TOTAL

Los resultados obtenidos luego de aplicar la lista de chequeo se reflejan en el Gráfico 3:

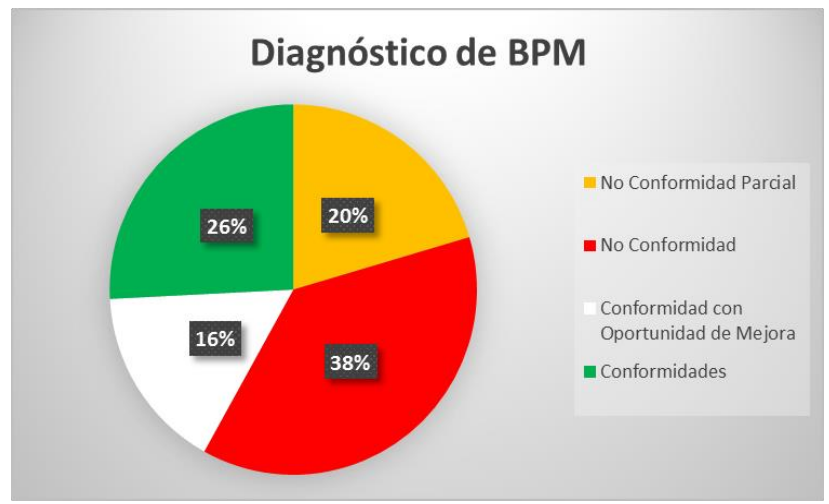

Gráfico 3. Resultado del diagnóstico de BPM en la empresa. 
En el gráfico anterior, se expresan los porcentajes de los resultados del diagnóstico de buenas prácticas de manufactura de la industria estudiada realizado a través de la lista de chequeo, encontrándose que las no conformidades o incumplimientos poseen el mayor porcentaje $38 \%$, las conformidades parciales un $20 \%$ y cumplimientos con oportunidades de mejora con un 16\%. Sobre estos ítems se trabajó los planes de manejo ambiental para conformar el Sistema de Gestión Ambiental basado en buenas prácticas de manufactura de la empresa, como se puede apreciar en la gráfica 4.

\section{Evaluación Ambiental}

\section{Diagnóstico de Residuos Sólidos}

\section{Producción de jugos}

En el proceso de producción de jugos se pudo identificar la generación de tres tipos de residuos: sólidos urbanos, Residuos tóxicos y peligrosos y Residuos especiales. Los residuos sólidos urbanos se pudieron evidenciar dentro del proceso de producción en la recepción de materia prima, envasado y almacenamiento de producto terminado. Los residuos que se encontraron fueron: Plástico, Cartón, Pallets de madera.

Residuos tóxicos y peligrosos se encontraron dentro del proceso de producción en la adición de ácido cítrico y ascórbico, filtración y lavado de equipos, debido a las sustancias químicas utilizadas en estos procesos. Se identificaron los siguientes residuos: Recipientes de ácido ascórbico, Recipientes de ácido cítrico, Filtros, Elementos de limpieza con ácido ascórbico y cítrico. Para el caso de residuos especiales, estos fueron identificados dentro del proceso productivo en la recepción y dosificación de materia prima y en el envasado. De este tipo de residuos se encontraron Tanques de 50 gal metálicos y Empaques de jugos tetrabrik.

\section{Producción de licores}

En el proceso de producción de licor que consiste en la fabricación de whisky, vodka, tequila y ron, se identificaron, los siguientes residuos: Residuos sólidos urbanos y residuos tóxicos y peligrosos. Los Residuos sólidos urbanos encontrados se ubican en los procesos de recepción de materia prima, envasado, etiquetado y almacenamiento de producto terminado, siendo: cartón, vidrio, plástico, pallets de madera

Residuos tóxicos y peligrosos se encontraron en los procesos de filtrado y de lavado de equipos identificándose: filtros con alcohol, recipientes con sosa cáustica, elementos de limpieza con sosa cáustica.

\section{Inventario de Residuos Sólidos}

En base a la entrevista realizada al área de producción, de laboratorio de calidad de la planta y al área administrativa, se obtuvieron los siguientes datos:

Tabla 2. Inventario de Residuos Sólidos 


\begin{tabular}{lll}
\hline Residuos & Unidades & Total \\
\hline Residuos Urbanos no Peligrosos & & \\
Plástico (fundas y botellas) & $\mathrm{kg} / \mathrm{mes}$ & 149 \\
Cartón & $\mathrm{kg} / \mathrm{mes}$ & 1100 \\
Papel & Resmas / mes & 10 \\
Pallets de madera & Unidades / mes & 4 \\
Vidrio & $\mathrm{kg} /$ mes & 1600 \\
Residuos Tóxicos y Peligrosos & & \\
Recipientes de ácido ascórbico & Costales / mes & 12 \\
Recipientes de ácido cítrico & Costales / mes & 13 \\
Filtros con alcohol & Unidades & 165 \\
Recipientes con sosa cáustica & Unidades & 20 \\
Recipiente de Ácido Nítrico al 63\% & Unidades / mes & 20 \\
Residuos Especiales & & \\
Tanques de 50 gal metálicos & Unidades / mes & 10 \\
\hline
\end{tabular}

\section{Diagnóstico de Efluentes Industriales}

En la fabricación de jugos se identificó la generación de efluentes industriales en los siguientes procesos: Dosificación de materia prima, Dilución, Filtrado, Pasteurizado, Envasado y etiquetado, lavado de maquinaria. Adicionalmente, aquellos productos que no cumplen las normas de calidad son abiertos y vaciados en un tanque para su reprocesamiento. En esta actividad se vierte parte del jugo fabricado que va a los canales de evacuación de efluentes. La producción de licores genera efluentes industriales en los siguientes procesos: Dosificación Envasado y etiquetado, Lavado de maquinaria. En el envasado, parte del licor se pierde en el control de volumen de las botellas pasando licor a las canaletas de evacuación de efluentes industriales.

Los vertidos de jugos y licores, no solo causan pérdidas económicas, sino que adicionalmente incrementan la Demanda Química de Oxígeno DQO y la Demanda Bioquímica de Oxígeno $\mathrm{DBO}_{5}$ de los efluentes, generando valores que sobrepasan la normativa ambiental del país. Este incumplimiento ambiental puede ser controlado a través de Buenas Prácticas de manufactura, antes de plantearse una planta de tratamiento de aguas residuales (PTAR) que podría resultar muy costosa y poner en riesgo la sostenibilidad de la empresa. Esta aplicación de BPM conduce a una mejora de la calidad del efluente a lo largo del proceso crítico detectado, es decir el envasado y etiquetado de jugos y licores, reduciendo pérdidas de producto terminado y mejorando la calidad ambiental de la empresa.

\section{Diagnóstico de Emisiones a la Atmósfera}

En las visitas de campo realizadas se constató la presencia de dos calderos y un generador eléctrico. El generador eléctrico no es utilizado más de 60 horas al semestre, por lo cual no 
requiere la realización de monitoreos semestrales, tal como dicta la legislación ecuatoriana, sin embargo, los calderos han sido monitoreados de manera semestral, incumpliendo la emisión de monóxido de carbono $\mathrm{CO}$ en el caldero 1. Al igual que en los efluentes líquidos, el disponer de un procedimiento de BPM que establezca mantenimiento, calibración e inyección de aire adecuada, los niveles de contaminación por CO presentarán cumplimiento legal.

\section{Diagnóstico de Ruido Ambiental}

La empresa ha determinado tres puntos de monitoreo para la evaluación del ruido ambiental generado por sus procesos productivos. Los resultados de las evaluaciones realizadas establecen el cumplimiento de la normativa ambiental vigente respecto al factor estudiado, por lo que no se requiere implantar nuevos controles, más allá de los existentes en la actualidad.

Sistema de Gestión Ambiental basado en buenas prácticas de manufactura

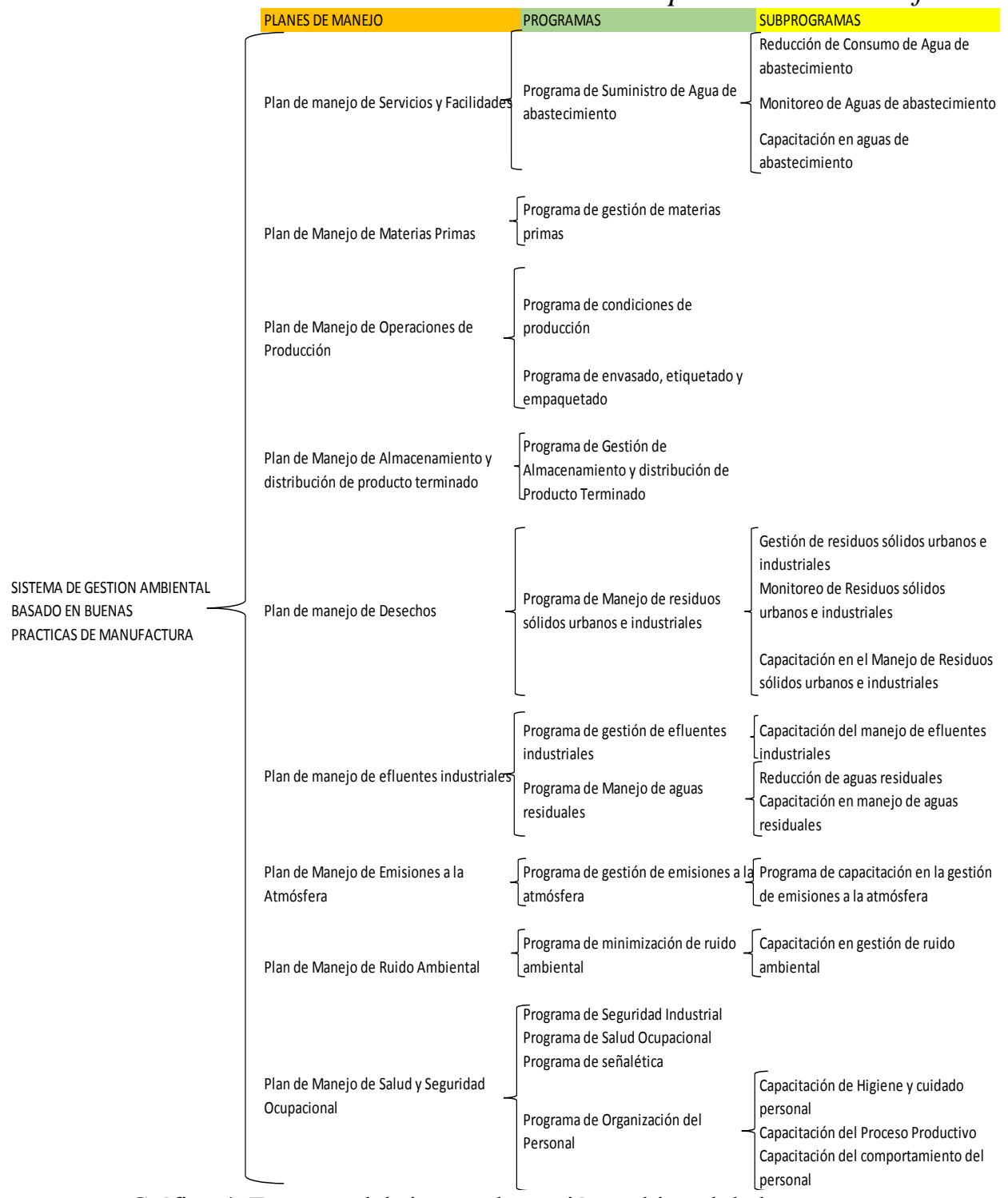

Gráfico 4. Esquema del sistema de gestión ambiental de la empresa

Este Sistema de Gestión Ambiental SGA ha sido elaborado en base tanto del análisis de 
Buenas Prácticas de Manufactura como de la evaluación ambiental de los procesos productivos, consta de planes que contienen programas y estos a su vez subprogramas, mismos que se encuentra detallados en el gráfico 4. Servirá de guía metodológica para el mejoramiento de la gestión ambiental de la empresa, lo que redundará en la disminución de costos operativos, optimización de insumos y mejora de la calidad de sus productos.

Los programas y subprogramas disponen de actividades y procedimientos para su cumplimiento, condensados en un Manual que, a más de las actividades y su fase de aplicación, dispone de indicadores de cumplimento, medios de verificación y la normativa legal aplicable. El Manual de aplicación del sistema de Gestión Ambiental basado en buenas prácticas de manufactura se ha representado en los gráficos 5,6 y 7 .

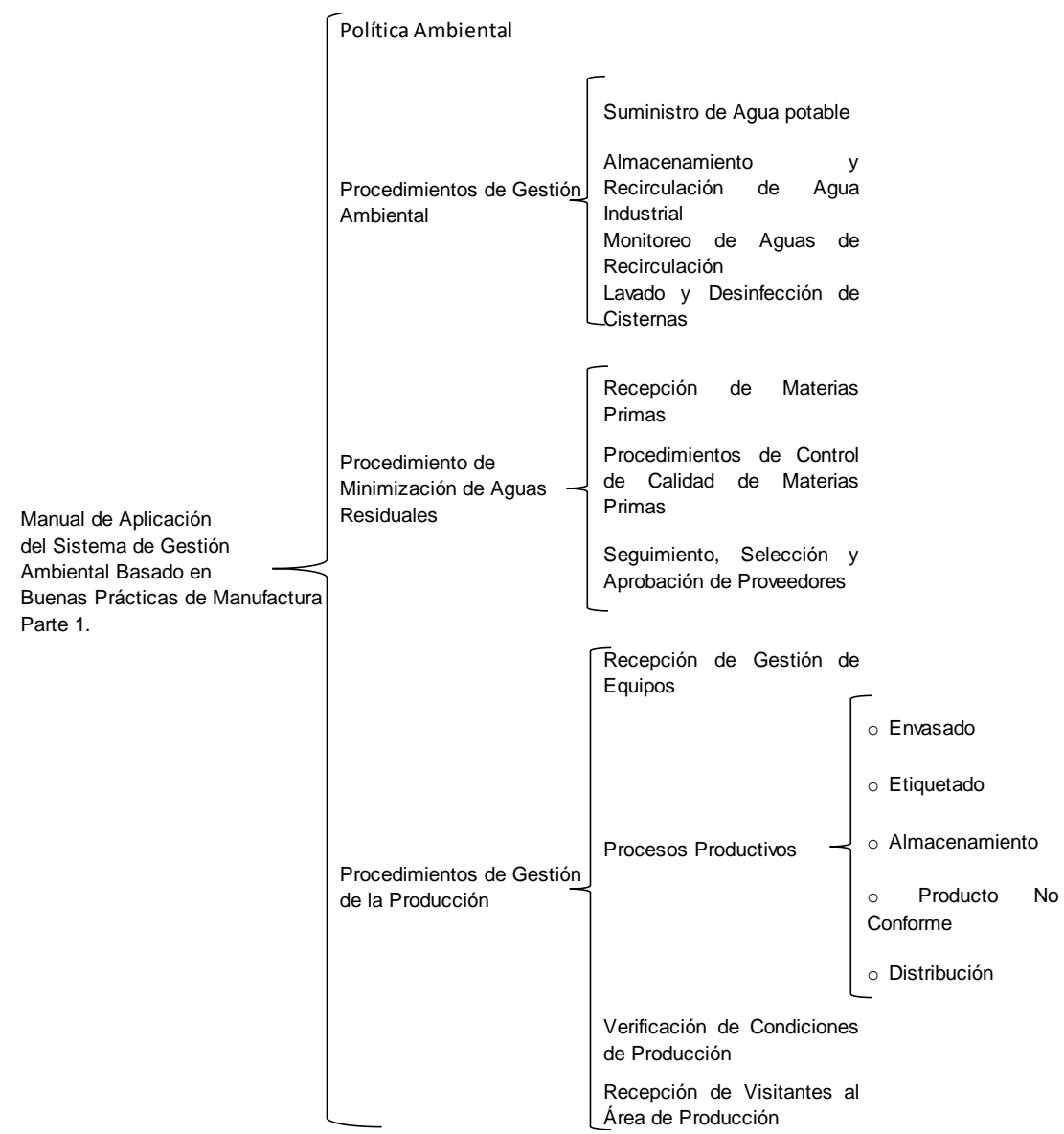

Grafico 5. Manual de Aplicación del SGA basado en BPM Parte 1. 


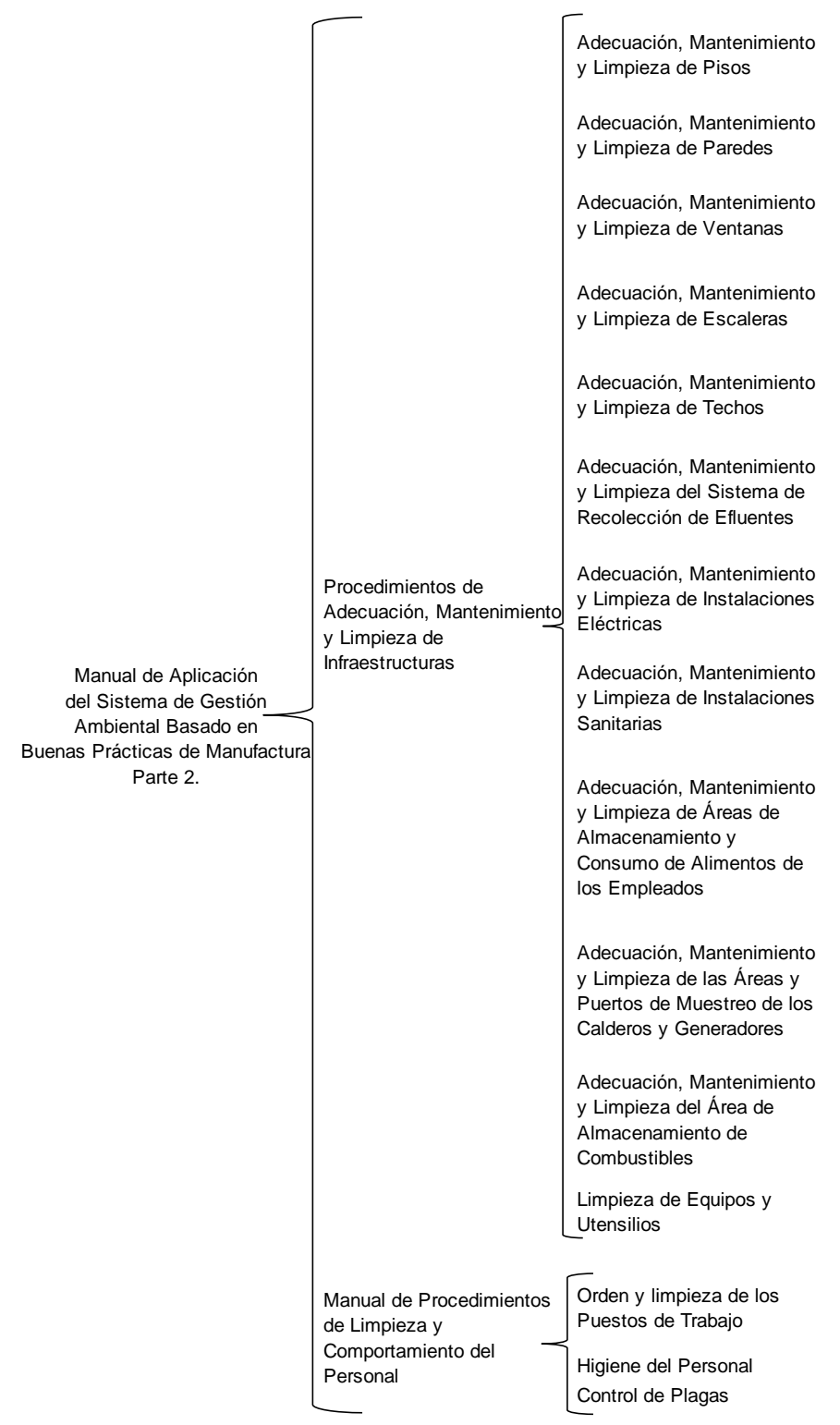

Grafico 6. Manual de Aplicación del SGA basado en BPM Parte 2 


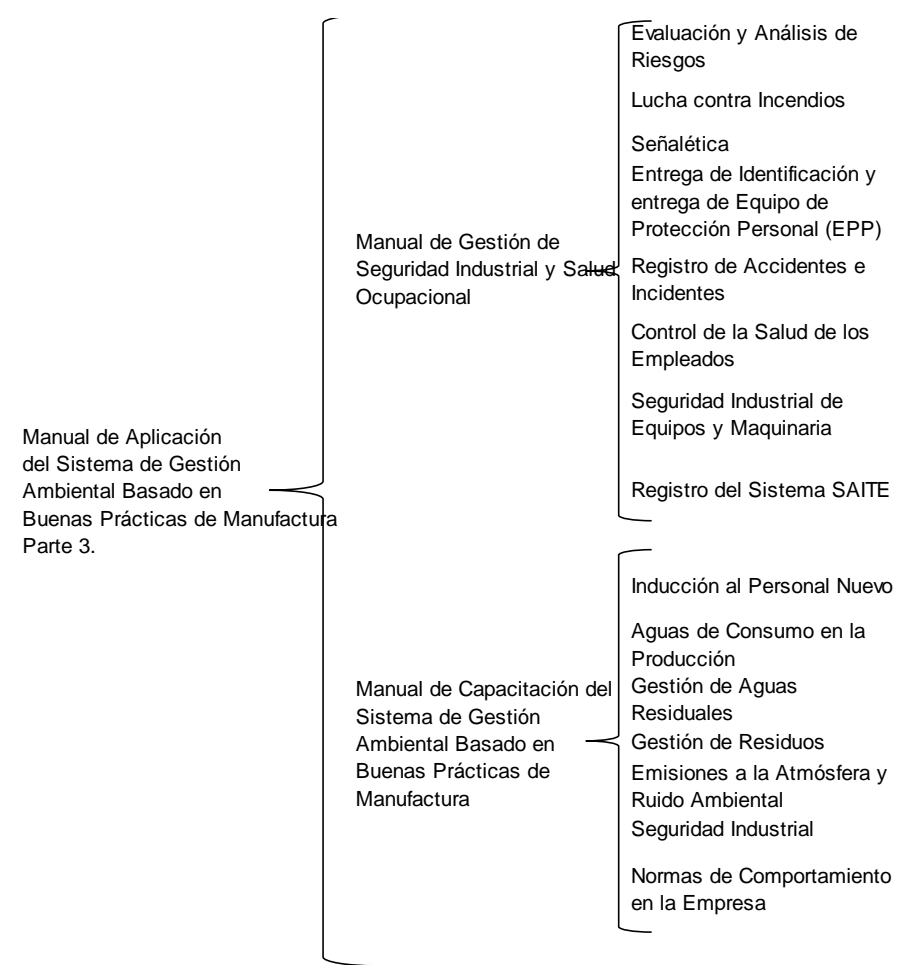

Grafico 7. Manual de Aplicación del SGA basado en BPM Parte 3

\section{Conclusiones y recomendaciones}

Los lineamientos que dan los Ministerios tanto de Industrias y Productividad como el de Ambiente, se correlacionan debido a que estos establecen normativas, reglamentos obligatorios y optativos, los cuales al conjugarlos permiten establecer parámetros específicos en el sentido de producción, sostenibilidad ambiental y seguridad y salud ocupacional, permitiendo a las industrias un mejor desempeño operacional, productivo y ambiental.

Los sistemas de Gestión Ambiental tradicionales, involucran una evaluación ambiental y de seguridad y salud ocupacional, sin embargo, no se contempla las buenas prácticas de manufactura. La correlación que se puede encontrar entre los sistemas de gestión ambiental tradicionales y las buenas prácticas de manufactura, radica en que los dos persiguen la mejora continua de los sistemas, sin embargo, la innovación de combinar estos criterios, mejora no solo la calidad del producto, sino también el aprovechamiento de materias primas, disminución de residuos y el buen manejo por parte del personal de los componentes productivos; adicionalmente, se resguarda la seguridad y salud ocupacional en la planta, garantizando productos competitivos y dando sostenibilidad a la empresa.

Los sistemas de gestión ambiental en las industrias, tradicionalmente, se basan en los diagnósticos levantados en función de parámetros ambientales exclusivamente, sin embargo, la innovación de la integración de buenas prácticas de manufactura al proceso de diagnóstico, crea una retroalimentación que optimiza la visión de la gestión productiva, aprovechando las materias primas y recursos económicos, así como el cumplimiento de la normativa ambiental vigente y el bienestar de los empleados. 
La Producción Más Limpia posee diferentes herramientas que son utilizadas en las industrias para desarrollar sistemas de gestión ambiental; sin embargo, las buenas prácticas de manufactura, tradicionalmente, no se toman en cuenta para el diagnóstico ambiental y posterior desarrollo de planes de manejo y programas de gestión ambiental. El aporte del presente trabajo, radica principalmente en la inclusión de esta herramienta de producción más limpia como un factor determinante en la elaboración del sistema de gestión.

La implementación de sistemas de gestión ambiental basados en buenas prácticas de manufactura, no solamente minimizan los impactos ambientales generados por las actividades productivas, su principal aporte es que se controla la generación de los efectos ambientales en el origen, facilitando el tratamiento y la disposición final de los efluentes y residuos generados en el proceso. La elaboración de jugos y licores debe caracterizarse por su asepsia. Las buenas prácticas de manufactura desarrollan competencias en el personal para el mejoramiento de la calidad de los productos, en función de los parámetros de higiene y de calidad establecidos. Al integrarlas al Sistema de Gestión Ambiental, el personal crea conciencia respecto a calidad y producción más limpia, haciéndolo más efectivo y aumentando la productividad de la empresa.

La metodología planteada, si bien es larga y meticulosa, permite obtener resultados satisfactorios, adecuados al sistema productivo particular de cada empresa, exactos en cuanto a diagnóstico y fáciles de comprender por los involucrados en el proceso productivo, aún a pesar de no haber sido ellos directamente los diseñadores de este sistema. Es fundamental, en esta metodología, tener conocimiento detallado de los diferentes procesos productivos, así como el funcionamiento en cuanto a producción, almacenamiento y distribución de los productos terminados, instalaciones y personal.

La metodología desarrollada en el presente trabajo es flexible para aplicarse en diferentes tipos de industrias alimenticias, ya que se dispone de la base de la matriz de los requerimientos establecidos en el Reglamento de ARCSA 067 - 2015, en el Anexo D. Esta metodología no se ve limitada por el tamaño de la industria y su producción, lo que le permite ser aplicada a pequeñas, medianas y grandes empresas. Para el sistema de gestión ambiental basado en buenas prácticas de manufactura, se deja a libre discreción de la empresa los responsables de los diferentes planes implementados en el sistema, así como la determinación de metas, las cuales se establecerán de acuerdo a los recursos, tiempo y personal que la empresa pueda dedicar a la implantación de este sistema; para ello es necesario el compromiso de la Alta Gerencia.

Para el diagnóstico ambiental de la empresa, de una lista de chequeo inicial de 260 ítems, se seleccionaron aquellos que se ajustaban en mayor medida al sistema productivo de la misma, esto se lo hizo para garantizar el acoplamiento de los requerimientos de Buenas Prácticas de Manufactura del Ministerio de Industrias de Ecuador, así como el cumplimiento de la normativa legal del Distrito Metropolitano de Quito. Se logró establecer, que si bien ambos sistemas persiguen diferentes objetivos, los dos velan por la minimización de impactos ambientales y el correcto funcionamiento operativo de la empresa.

De la evaluación realizada, se puede concluir que una de las debilidades de la empresa es la capacitación al personal, así como instrucciones específicas y asignaciones para las diferentes 
actividades laborales de los empleados, lo que no permite la especialización de los mismos generando deficiencias operativas, sobre todo en buenas prácticas de manufactura.

El sistema de gestión ambiental basado en buenas prácticas de manufactura, contempla una estructura conformada por planes, programas, subprogramas, manuales y procedimientos, lo que lo hace comprensible para cualquier persona que desee ponerlo en práctica, de tal forma que con una mínima capacitación por parte de la empresa, se garantice la continuidad del sistema propuesto. La flexibilidad de este sistema permitirá la aplicación e implementación del mismo en otro tipo de empresas. A lo largo del sistema de gestión se plantearon indicadores y medios de verificación para las actividades sugeridas, de tal manera que dichas actividades reflejen cumplimiento y mejora continua en el sistema implementado. Se procuró que la mayoría de los indicadores sean cuantitativos, sin embargo, en muchas actividades se vio la necesidad de plantear indicadores cualitativos, que no por ello son menos importantes que los primeros.

Los procedimientos establecidos en los manuales del sistema de gestión ambiental basado en buenas prácticas de manufactura dan cumplimiento a la normativa ambiental vigente, así como a los requerimientos operativos necesarios para las buenas prácticas de manufactura, sin embargo, estos procedimientos pueden ser revisados por la empresa y acoplados a las necesidades y disponibilidad de recursos. Igualmente, los manuales dejan a buen criterio de la empresa la designación de responsables y recursos para el cumplimiento de dichos procedimientos. El sistema es abierto, por lo cual la empresa tiene la oportunidad de modificar los indicadores estableciendo medios de verificación que permitan comprobar que la actividad se está cumpliendo adecuadamente.

La apertura y colaboración por parte de la Alta Gerencia de la empresa, es indispensable a la hora de desarrollar la metodología planteada, ya que existe información restringida que fue fundamental el momento de diagnosticar los parámetros y requerimientos previstos para el desarrollo del sistema de gestión ambiental basado en buenas prácticas de manufactura. Esta concienciación de la Alta Gerencia de la empresa, implica un total compromiso en la asignación de recursos destinados a la implementación del sistema propuesto a sabiendas que redundará en ganancias efectivas tanto a nivel productivo como financiero.

Del análisis de estudios realizados previamente, tanto para gestión ambiental como para buenas prácticas de manufactura, fue posible determinar que las empresas grandes y prósperas han basado parte de su desarrollo y éxito empresarial en la implementación independiente de los sistemas de gestión utilizados en el presente estudio, por lo que la combinación de ambos conceptos permitirá a la empresa un mejor manejo de sus recursos financieros dando lugar a su expansión en un futuro cercano. La empresa tiene gran potencial productivo y de calidad, lo que conjuntamente con la aplicación de este sistema va a redundar en una mejor utilización de tiempos, espacios y equipos, haciendo a la empresa más productiva y garantizando la estabilidad laboral de los trabajadores.

\section{Bibliografía}

Acuerdo ministerial 026 del 12 de mayo de 2008. Registro Oficial 334 
Acuerdo Ministerial 097 del 04 noviembre del 2015. Registro Oficial 387.

Acuerdo Ministerial 141 del 10 de julio de 2015. Registro Oficial 540.

Acuerdo Ministerial No. 142 del Ministerio del Ambiente. "Listados Nacionales de Sustancias Químicas Peligrosas, Desechos Peligrosos y Desechos Especiales”, R. O. No. 856 del 21 de diciembre de 2012.

Agencia Nacional de Regulación, Control y Vigilancia Sanitaria (ARCSA) (2016). "Normativa para Buenas Prácticas de Manufactura". Alimentos.

Decreto ejecutivo 2393 Reglamento de seguridad y salud de los trabajadores y mejoramiento del medio ambiente de trabajo

Ministerio de Fomento (s.f.). Puertos del Estado. Calidad (Nivel 1). Tomado de: http://www.apmarin.com/download/691_cal1.pdf. El 15/02/2016 a las 0h22

Ministerio de Industrias y Productividad (2016). Reglamento de Buenas Prácticas para alimentos procesados. Sello Hace bien.

Ministerio del Ambiente (2016). Punto Verde. Tomado de: http://www.ambiente.gob.ec/puntoverde/. El 01/03/2016 a las 20h02.

NTE INEN 1108:2011, agua potable.

NTE INEN 2 266:2010, transporte, almacenamiento y manejo de materiales peligrosos. Requisitos

NTE INEN 2 288:2000, productos químicos industriales peligrosos. Etiquetado de precaución. Requisitos

NTE INEN 2841, del marzo de 2014 Esta mal el orden de jerarquía

NTE INEN ISO 3864-1, colores, señales y símbolos de seguridad. 2013

NTP 481 (1998). Orden y Limpieza de Lugares de Trabajo. Instituto Nacional de Seguridad e Higiene en el Trabajo. España

OHSAS 18001 (2007). Sistemas de Gestión de Seguridad Industrial y Salud Ocupacional: Requisitos

OMS, (2007). CODEX ALIMENTARIUS. Quinta edición

Ordenanza Municipal 332 DMQ. Gestión integral de residuos sólidos del DMQ. 2010 
Ordenanza Municipal 404 DMQ. Expide las normas Técnicas para la Aplicación de la Codificación del Título V, “del Medio Ambiente”, Libro Segundo, del Código Municipal para el Distrito Metropolitano de Quito.

Resolución ARCSA-DE-067-2015-GGG La Dirección Ejecutiva de la Agencia Nacional de Regulación, Control y Vigilancia Sanitaria. Dado en Quito D.M., a los 21 días de diciembre de 2015

Torres M. 2012. “Análisis de Peligros y Puntos Críticos de Contra en el Complejo Industrial Licorero del Centro C.A. Universidad Simón Bolívar". Decanato de estudios Profesionales. Coordinación de Ingeniería Química. Venezuela. 